VERSION JULY 25, 2018

Preprint typeset using LATEX style AASTeX6 v. 1.0

\title{
X-RAY EMISSION FROM THE NUCLEAR REGION OF ARP 220
}

\author{
Alessandro Paggi ${ }^{1}$, Giuseppina Fabbiano ${ }^{1}$, Guido Risaliti ${ }^{1,2}$, Junfeng Wang $^{3}$, Margarita Karovska ${ }^{1}$, Martin Elvis ${ }^{1}$, W. Peter Maksym ${ }^{1}$, \\ JonATHAN McDowell ${ }^{1}$ and JAY GALLAGHER ${ }^{4}$ \\ ${ }^{1}$ Harvard-Smithsonian Center for Astrophysics, 60 Garden St, Cambridge, MA 02138, USA: apaggi@ @fa.harvard.edu \\ ${ }^{2}$ INAF-Arcetri Observatory, Largo E, Fermi 5, I-50125 Firenze, Italy \\ ${ }^{3}$ Department of Astronomy and Institute of Theoretical Physics and Astrophysics, Xiamen University, Xiamen, China 361005 \\ ${ }^{4}$ Department of Astronomy, University of Wisconsin, Madison, WI 53706-1582, USA
}

\begin{abstract}
We present an imaging and spectral analysis of the nuclear region of the ULIRG merger Arp 220, using deep Chandra-ACIS observations summing up to $\sim 300 \mathrm{ks}$. Narrow-band imaging with sub-pixel resolution of the innermost nuclear region reveals two distinct Fe-K emitting sources, coincident with the infrared and radio nuclear clusters. These sources are separated by 1' $(\sim 380 \mathrm{pc})$. The X-ray emission is extended and elongated in the eastern nucleus, like the disk emission observed in millimeter radio images, suggesting starburst dominance in this region. We estimate Fe-K equivalent width $\gtrsim 1 \mathrm{keV}$ for both sources, and observed 2-10 keV luminosities $\sim 2 \times 10^{40} \mathrm{erg} \mathrm{s}^{-1}(\mathrm{~W})$ and $\sim 3 \times 10^{40} \mathrm{erg} \mathrm{s}^{-1}$ (E). In the 6-7 keV band the emission from these regions is dominated by the $6.7 \mathrm{keV} \mathrm{Fe} \mathrm{xxv} \mathrm{line,} \mathrm{suggesting} \mathrm{contribution} \mathrm{from} \mathrm{collisionally} \mathrm{ionized} \mathrm{gas.} \mathrm{The} \mathrm{thermal}$ energy content of this gas is consistent with kinetic energy injection in the interstellar medium by Type II SNe. However, nuclear winds from hidden AGN $\left(v \sim 2000 \mathrm{~km} \mathrm{~s}^{-1}\right)$ cannot be excluded. The $3 \sigma$ upper limits on the neutral Fe-K $\alpha$ flux of the nuclear regions correspond to intrinsic AGN 2-10 keV luminosities $<1 \times 10^{42} \mathrm{erg} \mathrm{s}^{-1}$ (W) and $<0.4 \times 10^{42} \mathrm{erg} \mathrm{s}^{-1}$ (E). For typical AGN SEDs the bolometric luminosities are $<3 \times 10^{43} \mathrm{erg} \mathrm{s}^{-1}$ (W) and $<8 \times 10^{43} \mathrm{erg} \mathrm{s}^{-1}(\mathrm{E})$, and black hole masses $<1 \times 10^{5} M_{\odot}(\mathrm{W})$ and $<5 \times 10^{5} M_{\odot}$ (E) for Eddington limited AGNs with a standard $10 \%$ efficiency.
\end{abstract}

Keywords: galaxies: active — galaxies: individual (Arp 220) — galaxies: ULIRG — galaxies: interactions — $\mathrm{X}$-rays: galaxies

\section{INTRODUCTION}

At a distance of $80 \mathrm{Mpc}$ (Kim \& Sanders 1998), Arp 220 (IC 4553/4) is both a merger, and the nearest Ultra-luminous IR Galaxy (ULIRG; Soifer et al. 1987; Sanders \& Mirabel 1996). Near IR high-resolution (0.1") NICMOS-HST imaging identifies the two nuclear regions of the merging galaxies, which are coincident with the two components of a double radio source (Baan \& Haschick 1995; Scoville et al. 1998). At a separation of 1" (380 pc at a distance of $\left.80 \mathrm{Mpc}^{1}\right)$, these nuclei are closer together than the nuclei of NGC 6240 ( 690 pc separation, Komossa et al. 2003), and therefore may be subject to even stronger gravitational interaction, leading to accretion on the nuclei of the merging galaxy (e.g., Governato et al. 1994; Mayer et al. 2007).

The presence of AGN emission in addition to intense star formation in the two nuclei has been debated. It is also not clear where this AGN emission may reside. AGN contribution to the bolometric luminosity between $\sim 5 \%$ and $\sim 25 \%\left(0.1-0.4 \times 10^{12} L_{\odot}\right)$ is suggested by the Spitzer Mid-IR spectrum of the central 8" region of Arp 220 (Veilleux et al. 2009; Nardini et al. 2010; more recent Herschel results (Rangwala et al. 2011) and modeling of the nuclear spectra (Contini 2013) agree with this conclusion. In the west nucleus of Arp 220 the presence of a maser (Aalto et al. 2009) and a rotating massive molecular disk (Downes \& Eckart 2007) suggests a massive nuclear black hole. Instead, IRAM-PdBI observations (König et al. 2012) showed in the eastern nucleus of Arp 220 an extended structure in the mm-CO gas emission, elongated in the NE-SW direction, and suggested a highly dust-obscured nucleus lying between the two NIR sources detected by Scoville et al. (1998). Recently, an analysis of 3.5 and $1.2 \mathrm{~mm}$ IRAM-PdBI data performed by Tunnard et al. (2015) provided evidence of significant chemical differences between the two nuclei. These authors argue for a significant AGN contribution to the W nucleus luminosity, and starburst dominance in the E nucleus. ALMA imaging at 350 and $696 \mathrm{GHz}$ (Scoville et al. 2015) suggested compact nuclear disks with masses of $\sim 4$ and $\sim 2 \times 10^{9} M_{\odot}$ for $\mathrm{W}$ and $\mathrm{E}$ nucleus, respectively, within a radius $\sim 70 \mathrm{pc}$. High-resolution VLA observations at 33 and $6 \mathrm{GHz}$

${ }^{1}$ In the following, we adopt the standard flat cosmology with $\Omega_{\Lambda}=0.69$ and $H_{0}=68 \mathrm{~km} \mathrm{~s}^{-1} \mathrm{Mpc}^{-1}$ (Planck Collaboration et al. 2016). 
by Barcos-Muñoz et al. (2015) showed double radio morphology. These authors estimated for both nuclei high hydrogen column densities $\sim 10^{25} \mathrm{~cm}^{-2}$, and the star formation rate surface densities among the most extreme measured for any star-forming system $\left(\sim 10^{4} M_{\odot} \mathrm{yr}^{-1} \mathrm{kpc}^{-2}\right)$, without any compelling evidence of an AGN dominating the nuclei emission at $33 \mathrm{GHz}$.

The M- $\sigma$ relation (e.g., Magorrian et al. 1998) has suggested that the evolution of galaxies and super-massive nuclear black holes are linked. Both the stellar population and the SMBH of a galaxy are thought to grow and evolve by merging of smaller gas-rich galaxies and their nuclear SMBHs (Di Matteo et al. 2005; Hopkins et al. 2008). During this process, the SMBH may be "buried" by thick molecular gas, which feeds the SMBH at high rates, causing the birth of an obscured Compton Thick (CT, Risaliti et al. 1999; Levenson et al. 2006) Active Galactic Nucleus (AGN). In this paper we re-examine the X-ray emission of Arp 220. A high resolution study of the archival 57 ks Chandra ACIS observation (obsid 869; Clements et al. 2002) measured the hard X-ray luminosity of the central AGN but had insufficient signal to noise ratio and spatial resolution to detect a line or to spatially untangle the complex central emission. The X-ray spectrum extracted from the central $\sim 3$ " region suggested hard continuum and $\sim 6.6 \mathrm{keV}$ Fe-K emission line. A subsequent XMM-Newton observation detected Fe-K line emission centered at $6.7 \mathrm{keV}$ with an equivalent width $\mathrm{EW} \sim 1.9 \mathrm{keV}$ (Iwasawa et al. 2005) suggesting highly photoionized, low-density gas illuminated by a hidden CT AGN. A reanalysis of the Chandra and XMM-Newton data (LaMassa et al. 2011) only managed to set an upper limit on the neutral Fe-K $\alpha$ emission at $6.4 \mathrm{keV}$.

Here we make use of new deep Chandra-ACIS data. Together with the archival observation, we reach a total exposure of $\sim 300 \mathrm{ks}$, which allows us to perform a study of the X-ray emission from the nuclear region of Arp 220 with unprecedented detail, by means of sub-pixel imaging of Chandra ACIS data in narrow spectral ranges. This technique has been used successfully to study crowded emission regions of nearby Seyferts (e.g. in NGC 4151 Wang et al. 2011a,b,c; Mrk 573, Paggi et al. 2012). Our new look at the nuclear region of Arp 220, has resulted in the discovery of two sources in the 6-7 keV Fe-K band, spatially coincident with the near-IR and radio positions. In Section 2 we present the data reduction procedures for the imaging and spectral analysis; in Section 3 we discuss and interpret our results; in Section 4 we then draw our conclusions. In the following analysis we fix the Galactic absorption to the value $N_{H, g a l}=3.9 \times 10^{20} \mathrm{~cm}^{-2}$.

\section{DATA REDUCTION}

Chandra observations used in this analysis are listed in Table 1. Level 2 event files were retrieved from the Chandra Data Archive $^{2}$ and reduced with the CIAO (Fruscione et al. 2006) 4.7 software and the Chandra Calibration Data Base (CALDB) 4.6.7, adopting standard procedures. After excluding time intervals of background flares exceeding $3 \sigma$ with the LC_SIGMA_CLIP task, we obtained a low-background total exposure time of $\sim 290 \mathrm{ks}$. The nucleus has no significant pile up, as measured by the CIAO PILEUP_MAP tool ${ }^{3}$.

\subsection{Imaging analysis}

Imaging analysis was performed without pixel randomization to take advantage of the telescope dithering in event positioning and with the sub-pixel event repositioning (SER) procedure ( $\mathrm{Li}$ et al. 2003). We used a pixel size 1/4 of 0.492", the native Chandra/ACIS detector pixel (see, e.g., Harris et al. 2004; Siemiginowska et al. 2007; Perlman et al. 2010; Wang et al. 2011a). Using the same Orion ACIS-S data as in the calibration of Li et al. (2003), we find a significant $\Delta=50 \%$ (improvement in PSF FWHM as defined in Li et al. 2003) from sub-pixel repositioning for an on-axis source at 6-7 keV $(\Delta=70 \%$ at $\sim 2 \mathrm{keV}$ because of the narrower PSF at larger energies). Most of the imaging improvement is from sub-pixel event repositioning (SER) procedure (Li et al. 2003) and without pixel randomization, to take advantage of the sampling of the PSF by the well characterized spacecraft dither motion. Because of the similarly 'peaked' inner PSF, this is similarly effective at 2 and $6 \mathrm{keV}$.

In the top panels of Figure 1 we show the central 5" region of Arp 220 as imaged by the three Chandra-ACIS observations in the broad $0.5-8 \mathrm{keV}$ band with native $0.492^{\prime \prime}$ pixel size. To merge the three exposures we first used the WAVDETECT task to identify point sources in the field of each observation (excluding the central region). We then used the REPROJECT_ASPECT task to modify the aspect solutions minimizing position differences between these sources and finally merged the re-aligned images with the REPROJECT_EVENTS task to reproject the event files to the deepest 16092 observation. The relative shifts between the observations is $\sim 0.5^{\prime \prime}$, comparable with the $0.6^{\prime \prime}$ Chandra astrometric uncertainty.

In the bottom panels of Figure 1 the same central 5" region is shown in the narrow Fe-K 6-7 keV band with sub-pixel binning of $1 / 4$ of the native pixel size and 3 pixel FWHM gaussian filter smoothing. The narrow-band images for the deeper observations - 16092 and 16093 - show two sources associated with the West (W) and East (E) Arp 220 nuclei separated by 1" ( 380 pc at the source distance), while in the shorter 00869 observation only the $\mathrm{W}$ nucleus is detected. The locations of these unique emission

\footnotetext{
2 http://cda.harvard.edu/chaser

${ }^{3}$ Pile-up occurs on X-ray CCDs for source with high flux levels, when two or more photons arrive within the same detector region within a single CCD frame integration time, and they are counted a single photon of higher energy(Davis 2001).
} 
regions strongly argue for an identification of these sources with the nuclei of the merging galaxies. On the same panels we also show in green the position of the Chandra PSF artifacts as obtained with MAKE_PSF_ASYMMETRY_REGION ${ }^{4}$ tool for the two nuclei. PSF asymmetries are expected in the north-west direction for Obs ID 869, and in north-east direction for Obs IDs 16092 and 16093 (due to different roll angles). The two nuclear sources are not affected by PSF asymmetries, but the extension in the northeast direction of the E source, shown in the deepest observation 16092, overlaps with the region of Chandra PSF asymmetry which, however, can account for only up to $10 \%$ of the counts of this feature. The resulting merged images are shown in Figure 2 in the 3-6 (left panel), and 6.4-6.7 (central panel) keV bands with sub-pixel binning 1/4 of the native pixel size. In addition, in the right panel of the same figure we show the 6.4-6.7 keV band image after continuum (3-6 keV) subtraction (see Sect. 2.2). These images are indicative of continuum and Fe xxv line emission. The regions of $\mathrm{W}$ and $\mathrm{E}$ nuclei considered for spectral extraction discussed in Section 2.2 are shown as white circles.

Using PSF simulations performed with Chandra Ray Tracer (ChaRT ${ }^{5}$, Carter et al. 2003) taking into account the source spectrum, exposure time and off-axis angle, we applied the Expectation through Markov Chain Monte Carlo (Esch et al. 2004; Karovska et al. 2005, 2007; Wang et al. 2014) PSF-deconvolution algorithm to the merged images of the narrow-band emission. This method yields a multi-scale image reconstruction specifically applicable to Poisson noise limited data.

As noted by Clements et al. (2002), the lack of USNO detected stars in Arp 220 field does not allow accurate absolute astrometry using field stars. However, a comparison between the merged 6-7 keV Chandra-ACIS image and the high-resolution $33 \mathrm{GHz}$ VLA observations (Barcos-Muñoz et al. 2015) shows a clear similarity in the morphology of the nuclei. In order to highlight the morphological similarity between the X-ray and the radio emission, we shifted the VLA $33 \mathrm{GHz}$ sources shifting the latter in the NE direction by 0.2" (compatible with the Chandra astrometric accuracy, Rots \& Budavári 2011) in order to match the position of the western radio lobe with the western X-ray nucleus, that appears less extended and allows therefore a more accurate positioning. The result of this registration procedure is shown in Figure 3. In both the X-ray image and the radio contours the W nucleus looks compact while the E nucleus appears somewhat extended. We performed a similar registration with radio contours of $150 \mathrm{MHz}$ LOFAR continuum and CO 2-1 IRAM PdBi images (Varenius et al. 2016), and with $2.6 \mathrm{~mm}$ continuum ALMA images (Scoville et al. 2017). Again we matched the western nuclei. We notice that the positions of western lobes in VLA $33 \mathrm{GHz}$, LOFAR $150 \mathrm{MHz}$, IRAM PdBi CO 2-1 and ALMA $2.6 \mathrm{~mm}$ images differ less $\sim 0.1^{\prime \prime}$, and therefore the use of these maps yields similar results. In all cases the deconvolved X-ray image shows a more compact emission from the $\mathrm{W}$ nucleus, and a more extended E nucleus with a morphology similar to that of the $33 \mathrm{GHz}, \mathrm{CO} 2-1$ and $2.6 \mathrm{~mm}$ radio emissions suggesting starburst dominance in this region. On the other hand, the E X-ray nucleus lies somewhat north of the E lobe observed in the 150 $\mathrm{MHz}$ continuum LOFAR image. The central peak corresponds with the X4 X-ray source from Clements et al. (2002). Since the registration of radio images to the X-ray data yields similar results, in the following we will compare to VLA $33 \mathrm{GHz}$ data.

Figure 4 compares the Chandra merged images in the $3-6 \mathrm{keV}$ continuum with the observed 6-7 keV band selected to represent both the $6.4 \mathrm{keV}$ emitted $\mathrm{Fe} \mathrm{K}-\alpha$ neutral line and the $6.7 \mathrm{keV} \mathrm{Fe} \mathrm{xxv} \mathrm{line.} \mathrm{The} \mathrm{3-6} \mathrm{keV} \mathrm{continuum} \mathrm{emission} \mathrm{centroid}$ lies in between the peaks of the nuclear line emission (although closer to the $\mathrm{W}$ nucleus). The 6-7 keV peak coincide with the NIR sources reported by Clements et al. (2002), and the $5 \mathrm{GHz}$ peaks. The $\mathrm{Fe} 6-7 \mathrm{keV}$ image suggests a more extended $\mathrm{E}$ nucleus emission with respect to the $\mathrm{W}$ one. The X1 source reported by Clements et al. (2002) in the first $57 \mathrm{ks}$ Chandra dataset coincides with the W nucleus, while the X4 source from the same study lies between the two nuclei and it is coincident with the central peak seen the PSF-deconvolved 6-7 keV image.

\subsection{Spectral analysis}

We attempted a spectral characterization of the emission, extracting 3-8 keV spectra from the two circular regions indicated in Figure 2. The two easternmost peaks shown in Figure 3 do not have enough counts to be studied separately (see Table 2) and we therefore selected a region that encompasses them both. Spectra were extracted with CIAO sPECEXTRACT task, applying a point-source aperture correction, binned to obtain a minimum of 5 counts per bin, and fitted employing the Cash statistic.

\subsection{AGN Model}

We first used a model typical of CT AGN emission (Levenson et al. 2006), comprising an absorption component fixed to the Galactic value $3.9 \times 10^{20} \mathrm{~cm}^{-2}$, a power-law and red-shifted gaussian $\mathrm{Fe}-\mathrm{K}$ line(s) with width fixed to $100 \mathrm{eV}$, plus possible additional Ar, S and Ca lines as reported by Iwasawa et al. (2005). We used both XSPEC (ver. 12.8.2 $2^{6}$ ) and SHERPA ${ }^{7}$ with identical results. The extracted spectra and the best-fit parameters are presented in Figure 5 and Table $2^{8}$, respectively. Spectra

\footnotetext{
${ }^{4}$ http://cxc.harvard.edu/ciao/ahelp/make_psf_asymmetry_region.html

5 http://cxc.harvard.edu/chart/

${ }^{6}$ https://heasarc.gsfc.nasa.gov/xanadu/xspec

${ }^{7}$ http://cxc.harvard.edu/sherpa

${ }^{8}$ In the following, errors correspond to the $1-\sigma$ confidence level for one parameter of interest.
} 
were extracted from nuclear regions shown in Figure 2, as well as in the entire central region of Arp 220 using a circular region of 4.5" radius centered at the coordinates of sources shown in Fig 2.

Due to low statistics, we first fit the power-law component excluding data from 6-7 keV energy range. We then froze the power-law spectral index so obtained and added a red-shifted gaussian Fe-K line with energy free to vary (columns 1, 4 and 7 of Table 2, model a). In order to evaluate the contribution from neutral and ionized Fe-K separately, we then froze the line rest-frame energy at $6.4 \mathrm{keV}$ (columns 2, 5 and 8 of Table 2, model b), and then added a second line with rest-frame energy frozen at 6.7 $\mathrm{keV}$ (columns 3, 6 and 9 of Table 2, model c) in order to evaluate the relative contribution of neutral and ionized Fe lines.

In each model, given the contamination of the continuum radiation by the extended emission (suggested by the spatial distributions of continuum photons in Figure 2) and the poor statistics, our estimate of the nuclear continuum luminosity is an upper limit, and the Fe-K EWs must be considered as lower limits.

Model (a) We added a single gaussian line to the power law. This leads to detections of Fe-K line features in both regions, with comparable EW (1.2 keV and $1.8 \mathrm{keV}$ in W and E region, respectively). As expected from the imaging, the Fe- $\mathrm{K}$ line is more luminous in the $\mathrm{E}$ nucleus $\left(0.8 \times 10^{40} \mathrm{erg} \mathrm{s}^{-1}\right)$ with respect to the $\mathrm{W}\left(0.3 \times 10^{40} \mathrm{erg} \mathrm{s}^{-1}\right)$. As already discussed, the observed 2-10 keV luminosities, $3.2 \times 10^{40} \mathrm{erg} \mathrm{s}^{-1}$ (E) and $2.0 \times 10^{40} \mathrm{erg} \mathrm{s}^{-1}(\mathrm{~W})$, should be considered as upper limits because of contamination. Given the rest-frame line energies, $\left(6.67 \pm 0.03\right.$ and $6.61_{-0.07}^{+0.06} \mathrm{keV}$ in $\mathrm{E}$ and $\mathrm{W}$ regions, respectively) Fe xxv appears to be dominant in the Fe-K range, with fluxes of $9.43_{-1.91}^{+2.17} \times 10^{-7} \mathrm{~cm}^{-2} \mathrm{~s}^{-1}$ and $4.01_{-1.59}^{+1.67}$ in $\mathrm{E}$ and $\mathrm{W}$ regions, respectively. In Figure 6 we show the value of the fit statistic as a function of the rest-frame line energy.

We compare our results with those of LaMassa et al. (2011), who extracted a spectrum of the entire central region of Arp 220 using the archival Chandra-ACIS observation (OBSID 00869). Using a circular 4.5" radius count extraction region we detect a line at $6.65 \pm 0.02 \mathrm{keV}$ with and equivalent width of $1.39_{-0.69}^{+1.44} \mathrm{keV}$, compatible with the XMM-Newton detection at $6.72_{-0.12}^{+0.10} \mathrm{keV}$ (Iwasawa et al. 2005).

Model b) To assess an upper limit to the neutral $\mathrm{Fe}-\mathrm{K} \alpha$ contribution, we held the line rest-frame energy fixed at $6.4 \mathrm{keV}$ (model b). Using a circular 4.5" radius extraction region like LaMassa et al. (2011), we confirm their results only being able to set an upper limit on the Fe-K $\alpha$ EW. We then performed the same spectral fitting in the $\mathrm{E}$ and $\mathrm{W}$ nuclear regions. As shown in Table 2, due to the lower statistics with respect to the 4.5" region, neutral $\mathrm{Fe}-\mathrm{K} \alpha$ is detected, although with lower significance with respect to Model a, with line fluxes of $1.78_{-1.27}^{+1.51}$ and $2.39_{-1.27}^{+1.59} \times 10^{-7} \mathrm{~cm}^{-2} \mathrm{~s}^{-1}$ for $\mathrm{E}$ and W nucleus, respectively.

Model c) Then, we added a second line with a rest-frame energy fixed at $6.7 \mathrm{keV}$ (model c) to try to evaluate the relative contribution of $\mathrm{Fe}-\mathrm{K} \alpha$ and $\mathrm{Fe}$ xxv lines. With this model the neutral iron line is detected only in the $\mathrm{W}$ nucleus region with a flux accounting $\sim 25 \% \pm 10 \%$ of the total line flux, while both in the E nuclear and in the central 4.5 " region we can only put an upper limit of $\sim 5 \times 10^{38} \mathrm{erg} \mathrm{s}^{-1}$ on the Fe-K $\alpha$ emission.

To further test the possible contribution of a neutral iron emission line to the 6-7 keV emission we see in Figure 4, we analyzed the archival XMM-Newton observations of Arp 220 discussed in Iwasawa et al. 2005. The data were reduced following a standard procedure, analogous to the one described by Iwasawa et al. (2005). The results are also in agreement: in a continuum plus single line model we obtain a best fit peak rest-frame energy $E=6.65 \pm 0.04 \mathrm{keV}$. However, if we fit the data with two lines with fixed peak rest-frame energies $E_{1}=6.4 \mathrm{keV}$ and $E_{2}=6.7 \mathrm{keV}$, we obtain the results shown in Figure 7: a neutral component accounting for up to $40 \%$ of the observed line flux cannot be ruled out at a $90 \%$ confidence level, which is compatible with the fluxes obtained with fixed $6.4 \mathrm{keV}$ lines.

\subsection{Thermal Model}

Finally, we investigate the possibility for the Fe-K lines to arise from thermal gas emission - possibly from merged supernova (SN) ejecta and stellar winds present during a starburst. To this end we fitted the 2-8 keV spectra with a collisionally ionized plasma component APEC and an intrinsic absorption component ZWABS at the source redshift (Model d), with element abundances both fixed at solar values and free to vary, and the results are reported in Table 2. The temperatures of the gas obtained from the fits are $\sim 5 \mathrm{keV}$, with column densities $\sim 5 \times 10^{22} \mathrm{~cm}^{-2}$ for the $\mathrm{W}$ and $\mathrm{E}$ regions, and $\sim 2 \times 10^{22} \mathrm{~cm}^{-2}$ in the central 4.5 " region. The metallicity are found to be $4.58_{-0.72}^{+1.29}, 1.14_{-0.44}^{+0.75}$ and $1.62_{-0.52}^{+0.59}$ in the $\mathrm{E}, \mathrm{W}$ and central $4.5^{\text {" }}$ regions, respectively. We notice, in particular, that the high metallicity obtained in the eastern nucleus can be due to chemical enrichment by Type II SNe in a starburst region, producing substantial $\alpha$-elements but a relatively small amount of iron. The Fe metallicity in this region is very large - about four times solar - but this can be the case for a region with intense star formation (e.g., Fabbiano et al. 2004). We note, however that this model is disfavored by the large statistics shown in Table 2 indicating that, if present, the thermal gas emission is likely to be sub-dominant with respect to harder emissions, possibly of nuclear origin. The results from model $\mathrm{d}$ must be therefore interpreted as upper limits on thermal gas emission. 


\section{DISCUSSION}

Making use of deep Chandra-ACIS observation and sub-pixel binning in narrow spectral bands we have detected three sources of emission in the 6-7 keV band, with the westernmost and easternmost ones coincident with the IR (W) and radio (E) nuclei of Arp 220 (see Figure 4). The spectral analysis (Section 2.2) showed Fe-K lines with large ( $21 \mathrm{keV}$ ) EWs and with the rest-frame line energies larger than $6.4 \mathrm{keV}$ of the neutral $\mathrm{Fe}-\mathrm{K} \alpha$ line, and compatible with $6.7 \mathrm{keV} F e$ xxv emission.

\subsection{CT AGN Models}

In this section we try to constrain the presence of a dual CT AGN in the Arp 220 nuclei. CT AGNs are characterized in the X rays by a hard high energy continuum, a "reflection" flat continuum in the $\sim 2-10 \mathrm{keV}$ range, and a high EW ( $\gtrsim 1) \mathrm{keV} 6.4$ Fe-K $\alpha$ line (e.g., Matt et al. 1997, 2000). Examples of this merger-driven evolution are given by the pairs of nuclei discovered in the $6.4 \mathrm{keV}$ Fe-K line with Chandra in the merger infrared (IR) luminous galaxy NGC 6240 (Komossa et al. 2003).

As for NGC 6240 (Komossa et al. 2003), Arp 220 is a highly disturbed system of galaxies engaged in a major merging interaction. The physical projected separation of the CT nuclei is $\sim 670 \mathrm{pc}$ in NGC 6240 and $\sim 380 \mathrm{pc}$ in Arp 220, suggesting that the latter may be in a more advanced stage of merging.

The spectral analysis of the individual $\mathrm{W}$ and $\mathrm{E}$ nuclei, results in the detection of Fe emission lines. The rest-frame energy of the line, however, is larger than the $6.4 \mathrm{keV}$ of the $\mathrm{K} \alpha$ line and suggests a contribution from $6.7 \mathrm{keV}$ shock-ionized $\mathrm{Fe} \mathrm{xxv}$ line. However, as shown in Figure 7, if we assume that both $6.4 \mathrm{keV}$ and $6.7 \mathrm{keV}$ lines are present in the spectrum, we obtain an acceptable fit to the XMM-Newton data which allow for $40 \% 6.4 \mathrm{keV}$ contribution. The statistics, however, does not allow us to disentangle the 6.4 and $6.7 \mathrm{keV}$ line contribution to the observed emission in Chandra data.

If we then consider that $40 \%$ of the Fe-K line flux we estimate from Chandra spectra is due to Fe-K $\alpha$ neutral $6.4 \mathrm{keV}$ emission line, the 2-10 keV emitted luminosities inferred from the Fe-K luminosities are $1.6 \times 10^{42} \mathrm{erg} \mathrm{s}^{-1}$ (E) and $0.5 \times 10^{42} \mathrm{erg} \mathrm{s}^{-1}$ (W). We note that these corrections are calibrated on "standard" obscured Seyfert galaxies, with an X-ray reflection efficiency of a few percent (Levenson et al. 2006). Hard X-ray observation of ULIRGs have demonstrated that on average this efficiency is much lower for these sources (Teng et al. 2009; Nardini \& Risaliti 2011). Consequently, the intrinsic X-ray luminosity of the two AGN detected here could be significantly higher. Considering the values for a standard reflection efficiency, the inferred X-ray luminosity is at least a factor of 3 higher than that expected from a pure starburst with the bolometric luminosity of Arp 220 (Ranalli et al. 2003).

The two nuclei have radio fluxes of 92.4 (E) and 114.6 (W) mJy at $4.7 \mathrm{GHz}$ (Barcos-Muñoz et al. 2015), and X-ray to optical slopes $\alpha(o x) \approx 2.75$. From the inferred $2-10 \mathrm{keV}$ emitted luminosities of the two nuclear sources we evaluate bolometric luminosities assuming typical AGN SEDs (Elvis et al. 1994, 2002), and the X-ray reflection efficiency of Seyfert galaxies. The estimated AGN bolometric luminosities, which should be regarded as lower limits, are $\sim 8.3 \times 10^{43} \mathrm{erg} \mathrm{s}^{-1}$ (E) and $~$ $2.5 \times 10^{43} \mathrm{erg} \mathrm{s}^{-1}(\mathrm{~W})$. These represent only a $\sim 1 \%$ of Arp 220 bolometric luminosity $\sim 6 \times 10^{45} \mathrm{erg} \mathrm{s}^{-1}$ (Sanders et al. 1988), confirming that, overall, the emission of Arp 220 is dominated by the starburst component. We note that the AGN luminosity evaluated with IR data by Veilleux et al. (2009); Nardini et al. (2010) is much higher than that inferred from our X-ray analysis, which is suggestive of heavy or nearly total obscuration. Lower limits on associated BH masses can be evaluated assuming Eddington limited accretion (with a standard $10 \%$ accretion rate to luminosity conversion efficiency), yielding $M \sim 5 \times 10^{5} M_{\odot}$ (E) and $\sim 1 \times 10^{5} M_{\odot}(\mathrm{W})$.

The lack of $6.4 \mathrm{keV}$ emission may result from a limited visibility of the inner torus surrounding the nuclear AGNs, allowing only the emission of ionized gas to be visible (Iwasawa et al. 2005). To test this possibility we produced a hardness ratio (HR) map of the central region of this source (Figure 8, right panel). This map has been obtained from the event maps in the soft (S) 0.3-2 keV and hard (H) 2-8 keV bands with sub-pixel binning 1/4 of the native pixel size, evaluating $H R=(H-S) /(H+S)$ and then applying a $3 \mathrm{X} 3$ pixel FWHM gaussian filter smoothing. The white contours indicate levels of HR from 0.1 to 0.8 with increments of 0.1 . We then binned the event maps using these contours, producing the HR binned map presented in the central panel of Figure 8. In this panel we superimpose in yellow the $33 \mathrm{GHz}$ VLA contours from Barcos-Muñoz et al. (2015) to highlight the "bridge" of $H R \sim 0.8$ that connects the two nuclei detected both in radio and X-ray (see Figure 3) and corresponding with $\mathrm{X} 4$ source reported by Clements et al. (2002). On the right panel of Figure 8 we present a significance map of the HR binned map, evaluated as the ratio between the uncertainty on the HR and the HR itself, showing that this "bridge" feature is significant at $8 \sigma$ level. We then produced simulated spectra, assuming a power-law spectrum with slope 1.8 (as appropriate for AGN emission) and, in addition to the Galactic absorption, an intrinsic absorption component at the source redshift, and evaluated the observed HR such spectra would yield. In this way we converted the binned HR map to the intrinsic absorption column map presented in the left panel of Figure 9, where the logarithmic values of $N_{H} / \mathrm{cm}^{-2}$ are presented, with overplotted in blue the 33 $\mathrm{GHz}$ VLA contours. On the right panel of Figure 9 we show the map of the corresponding error on $\log \left(N_{H} / \mathrm{cm}^{2}\right)$ evaluated from the uncertainty on the HR. We see that in the region connecting the two nuclei we reach $N_{H} \sim 10^{22.5} \mathrm{~cm}^{2}$, similar to that 
obtained from the spectral fitting with model d, but about four order of magnitudes lower than the value of $N_{H_{2}}=2.6 \times 10^{26} \mathrm{~cm}^{2}$ reported by Scoville et al. (2017) for the W nucleus, indicating again that the AGN contribution to X-ray emission represents a sub-dominant component with respect to star formation activity (see next section).

\subsection{Star Formation Activity}

The nuclear region of Arp 220 is site of an intense star formation with SFR $340 M_{\odot} \mathrm{yr}^{-1}$ (Baan 2007; Barcos-Muñoz et al. 2015). The X-ray expected luminosity from X-ray binaries, evaluated using the correlation between the galaxy $2-10 \mathrm{keV}$ luminosity and the SFR provided by the Chandra survey of LIRGs (Lehmer et al. 2010), exceeds the observed luminosity by one order of magnitude. It is however possible, as suggested by the previous discussion, that X-ray binaries in Arp 220 are located in compact star-forming regions buried under thick absorption columns with $N_{H}>>10^{23} \mathrm{~cm}^{-2}$ that dims the emission in the 2-10 $\mathrm{keV}$ band (Lehmer et al. 2010).

As discussed before, the Fe Xxv emission may be related with the thermal gas from merged SN ejecta and stellar winds present during a starburst, as exemplified by the extended Fe xxv regions of the merger-dual CT AGN NGC 6240 Wang et al. 2014). Extended Fe xxv line emission is also observed in other well-known starburst galaxies like NGC 253 and M82 (e.g., Pietsch et al. 2001; Mitsuishi et al. 2011), the merger-dual CT AGN NGC 6240 Wang et al. 2014, and the integrated spectrum of a number of LIRG/ULIRG systems (Iwasawa et al. 2009), suggesting the existence of similar high temperature plasma.

According to the starburst-driven superwind model, a hot gas bubble of internally shocked wind material with a temperature of several keV forms in the region of intense star formation (Chevalier \& Clegg 1985; Suchkov et al. 1994; Strickland \& Heckman 2007); this hot gas eventually flows outward as a high-speed (few $1000 \mathrm{~km} \mathrm{~s}^{-1}$ ) wind. As a comparison, we note that the observed diffuse hard X-ray emission in Arp 220 has a luminosity one order of magnitude higher than that observed in the classic superwind system M82 $\left(L_{2-10} \mathrm{keV}=4 \times 10^{39} \mathrm{erg} \mathrm{s}^{-1}\right.$; Strickland \& Heckman 2007). Following the calculation of Wang et al. (2014) we can evaluate the shock velocity $v=\sqrt{16 k T / 3 \mu}$ (where $\mu$ is the mean mass per particle and $k$ is Boltzmanns constant) from the observed gas temperature $\sim 5 \mathrm{keV}$, obtaining $v \simeq 2000 \mathrm{~km} \mathrm{~s}^{-1}$. Such velocities are unlikely to be due to shocks induced by merging process of galaxies that collide with velocities of the order of hundreds of $\mathrm{km} \mathrm{s}^{-1}$ even in the case of direct collision systems (Braine et al. 2003), and they are as well larger than the velocity estimated from the CO line width of $500-600 \mathrm{~km} \mathrm{~s}^{-1}$ (Sakamoto et al. 2008; Scoville et al. 2017).

To further investigate whether the thermal energy content of the hot gas could be powered by thermalization of SNe shocks, we compare the thermal gas energy with the kinetic energy input from the SNe during the starburst. Using the Fe xxv line emission as a tracer for the extent of the hottest thermal component, and following the method of Richings et al. (2010), from the emission measure we obtain - assuming a filling factor of $1 \%$ - the hot gas masses of $2.6 \times 10^{5} M_{\odot}$ and $6.6 \times 10^{5} M_{\odot}$ for the W and E nuclei, respectively. For solar abundances this yields an iron mass of $6.4 \times 10^{2} M_{\odot}$ and $1.7 \times 10^{3} M_{\odot}$ for the $\mathrm{W}$ and E nuclei, respectively. From the temperatures we get from the spectral fit we obtain a thermal energy of $E_{t h, W}=1.3 \times 10^{55} \mathrm{erg}$ and $E_{t h, E}=3.1 \times 10^{55} \mathrm{erg}$, for the $\mathrm{W}$ and $\mathrm{E}$ nuclei, respectively. Assuming a SN rate for Arp 220 of $4 \mathrm{yr}^{-1}$ (Lonsdale et al. 2006) and that 10\% of the kinetic energy input ( $10^{51} \mathrm{erg}$ per SN) is converted into thermal energy of the hot gas (Chevalier \& Clegg 1985; Thornton et al. 1998), we

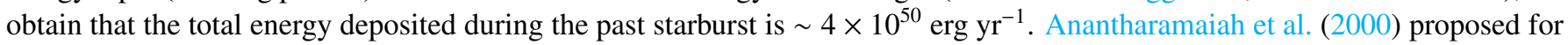
Arp 220 a star formation model consisting of multiple starbursts of very high SFR $\left(\sim 10^{3} M_{\odot} \mathrm{yr}^{-1}\right)$ and short duration $\left(\sim 10^{5} \mathrm{yr}\right)$. With this starburst period we obtain that the gas thermal energy is comparable with the kinetic energy input from the SNe. On the other hand, the cooling time of this thermal gas is $t_{\text {cool }}=E_{t h} / L_{x} \sim 50 \mathrm{Myr}$, which is far larger than the starburst duration, allowing the X-ray bright phases to survive once the starburst ceases. Note that such a short starburst can also explain the lack of X-ray point sources, that with a SFR $\left(\sim 10^{3} M_{\odot} \mathrm{yr}^{-1}\right)$, would attain a luminosity $\sim 10^{42} \mathrm{erg} \mathrm{s}^{-1}$ (Mineo et al. 2012). We can also compare the iron yield SNe over the starburst with the iron mass evaluated from the spectral fitting of the thermal gas emission. Using an ejected iron mass per Type II SN of $8.4 \times 10^{-2} M_{\odot}$ (Iwamoto et al. 1999), a SN of $4 \mathrm{yr}^{-1}$ (Lonsdale et al. 2006) over a starburst of $10^{5} \mathrm{yr}$ would yield $\sim 3.4 \times 10^{4} M_{\odot}$ of iron, more than enough to account for the iron mass of $1.7 \times 10^{3} M_{\odot}$ and $6.4 \times 10^{2} M_{\odot}$ evaluated before for the $\mathrm{E}$ and $\mathrm{W}$ nuclei, respectively. On the other hand, a continuous SFR of $350 M_{\odot} \mathrm{yr}^{-1}$ over a period of $\sim 1.5 \times 10^{7} \mathrm{yr}$ (Anantharamaiah et al. 2000) would yield a SNe kinetic energy input exceeding the gas thermal energy by two order of magnitudes and a cooling time about three time the starburst duration, with a yield of $\sim 3.4 \times 10^{6} M_{\odot}$ of iron.

These estimates demonstrate that the observed diffuse thermal gas traced by the highly ionized iron line emission in Arp 220 is consistent with being heated by SNe shocks in the starburst, although high velocity winds from hidden AGNs may also be present.

\section{CONCLUSIONS}

We presented an imaging and spectral analysis of the nuclear region of the ULIRG merger Arp 220 which make use of deep Chandra-ACIS observations that sum to $~ 300 \mathrm{ks}$. Narrow-band spectral imaging with sub-pixel resolution of the innermost nuclear region reveals two Fe-K emitting sources, spatially coincident with the infrared and radio emitting nuclear clusters, and 
separated by 1" ( $\sim 380 \mathrm{pc}$ at a distance of $\sim 80 \mathrm{Mpc})$. We estimate Fe-K equivalent width $\sim 1 \mathrm{keV}$ or possibly greater for both sources, and observed 2-10 keV luminosities $L_{X} \sim 2 \times 10^{40} \mathrm{erg} \mathrm{s}^{-1}(\mathrm{~W})$ and $\sim 3 \times 10^{40} \mathrm{erg} \mathrm{s}^{-1}$ (E).

In the narrow 6-7 keV band the emission from these regions is dominated by the $6.7 \mathrm{keV} F e \mathrm{xxv}$ emission line, suggesting a contribution from collisionally ionized gas or starburst regions (see also Iwasawa et al. 2005). The nuclear regions appear to be filled with a thermal gas at $\sim 5 \mathrm{keV}$ whose energetic content can be accounted for by kinetic energy injection in the interstellar medium by Type II SNe. As NGC 6240 (Wang et al. 2014), the thermal gas surrounding the nuclei and responsible for the hard $\mathrm{X}$-ray emission has a thermal energy comparable with the kinetic energy injected in the surrounding medium by Type II SNe in a short $\left(\sim 10^{5} \mathrm{yr}\right)$ starburst episode.

The X-ray emission from the eastern nucleus appears morphologically coincident with the disk emission as mapped by $2.6 \mathrm{~mm}$ continuum ALMA and CO 2-1 IRAM PdBi data, suggesting starburst dominance in this region. However, Chandra data allow us to constrain the contribution from (possibly dust-obscured) AGNs. In fact, our analysis of the XMM-Newton data confirms the presence of $\mathrm{Fe} \mathrm{xxv}$ emission line but allows $40 \%$ of the narrow-band emitted flux form the neutral $6.4 \mathrm{keV}$ line. Based on the $\mathrm{Fe}-$ $\mathrm{K}$ detections, we infer lower limits on the bolometric luminosity of the AGNs in Arp 220 of $8.3 \times 10^{43} \mathrm{erg} \mathrm{s}^{-1}$ for the E nucleus, and $\sim 2.5 \times 10^{43} \mathrm{erg} \mathrm{s}^{-1}$ for the $\mathrm{W}$ nucleus. These are a few percent of the total ULIRG bolometric luminosity, confirming that overall the emission of this source is dominated by the starburst component, as estimated from the mid-IR spectroscopy (Veilleux et al. 2009; Nardini et al. 2010). Our results are consistent with previous multi-wavelength indications of nuclear activity in Arp 220 (see Section 1), and strengthen the evolutionary association of merging and nuclear activity in galaxies (e.g., Hopkins et al. 2008; Van Wassenhove et al. 2012).

These results have only been possible because of the unmatched Chandra-ACIS spatial resolution coupled with the use of sub-pixel imaging in narrow spectral bands, which allow us to perform narrow-band, high resolution imaging that gives us a clear picture of the nuclear surroundings.

We acknowledge useful comments and suggestions by our anonymous referee. This work is supported by NASA grant G0415107X (PI. Fabbiano). JW acknowledges support from National Key Program for Science and Technology Research and Development 2016YFA0400702, and the NSFC grants 11473021,11522323. We acknowledge support from the CXC, which is operated by the Smithsonian Astrophysical Observatory (SAO) for and on behalf of NASA under Contract NAS8-03060. This research has made use of data obtained from the Chandra Data Archive, and software provided by the CXC in the application packages CIAO and Sherpa. This research has made use of Iris software provided by the US Virtual Astronomical Observatory, which is sponsored by the National Science Foundation and the National Aeronautics and Space Administration.

\section{REFERENCES}

Aalto, S., Wilner, D., Spaans, M., et al. 2009, A\&A, 493, 481

Anantharamaiah, K. R., Viallefond, F., Mohan, N. R., Goss, W. M., \&

Zhao, J. H. 2000, ApJ, 537, 613

Baan, W. A., \& Haschick, A. D. 1995, ApJ, 454, 745

Baan, W. A. 2007, IAU Symposium, 242, 437

Batejat, F., Conway, J. E., Rushton, A., et al. 2012, A\&A, 542, L24

Barcos-Muñoz, L., Leroy, A. K., Evans, A. S., et al. 2015, ApJ, 799, 10

Braine, J., Davoust, E., Zhu, M., et al. 2003, A\&A, 408, L13

Carter, C., Karovska, M., Jerius, D., Glotfelty, K., \& Beikman, S. 2003,

Astronomical Data Analysis Software and Systems XII, 295, 477

Chevalier, R. A., \& Clegg, A. W. 1985, Nature, 317, 44

Clements, D. L., McDowell, J. C., Shaked, S., et al. 2002, ApJ, 581, 974

Contini, M. 2013, MNRAS, 429, 242

Davis, J. E. 2001, ApJ, 562, 575

Di Matteo, T., Springel, V., \& Hernquist, L. 2005, Nature, 433, 604

Downes, D., \& Eckart, A. 2007, A\&A, 468, L57

Elvis, M., Wilkes, B. J., McDowell, J. C., et al. 1994, ApJS, 95, 1

Elvis, M., Risaliti, G., \& Zamorani, G. 2002, ApJL, 565, L75

Esch, D. N., Connors, A., Karovska, M., \& van Dyk, D. A. 2004, ApJ, 610, 1213

Fabbiano, G., Baldi, A., King, A. R., et al. 2004, ApJL, 605, L21

Fruscione, A., McDowell, J. C., Allen, G. E., et al. 2006, Proc. SPIE, 6270

Gilli, R., Comastri, A., \& Hasinger, G. 2007, A\&A, 463, 79

Governato, F., Colpi, M., \& Maraschi, L. 1994, MNRAS, 271

Harris, D. E., Mossman, A. E., \& Walker, R. C. 2004, ApJ, 615, 161
Hopkins, A. M., McClure-Griffiths, N. M., \& Gaensler, B. M. 2008, ApJL, 682, L13

Iwamoto, K., Brachwitz, F., Nomoto, K., et al. 1999, ApJS, 125, 439

Iwasawa, K., Matt, G., Guainazzi, M., \& Fabian, A. C. 2001, MNRAS, 326,894

Iwasawa, K., Sanders, D. B., Evans, A. S., et al. 2005, MNRAS, 357, 565

Iwasawa, K., Sanders, D. B., Evans, A. S., et al. 2009, ApJL, 695, L103

Karovska, M., Schlegel, E., Hack, W., Raymond, J. C., \& Wood, B. E. 2005, ApJL, 623, L137

Karovska, M., Carilli, C. L., Raymond, J. C., \& Mattei, J. A. 2007, ApJ, 661, 1048

Kim, D.-C., \& Sanders, D. B. 1998, ApJS, 119, 41

Komossa, S., Burwitz, V., Hasinger, G., et al. 2003, ApJL, 582, L15

König, S., García-Marín, M., Eckart, A., Downes, D., \& Scharwächter, J. 2012, ApJ, 754, 58

LaMassa, S. M., Heckman, T. M., Ptak, A., et al. 2011, ApJ, 729, 52

Levenson, N. A., Heckman, T. M., Krolik, J. H., Weaver, K. A., \& Życki, P. T. 2006, ApJ, 648, 111

Lehmer, B. D., Alexander, D. M., Bauer, F. E., et al. 2010, ApJ, 724, 559

Li, J., Kastner, J. H.,Prigozhin, G. Y., \& Schulz, N. S. 2003, ApJ, 590, 586

Lonsdale, C. J., Diamond, P. J., Thrall, H., Smith, H. E., \& Lonsdale, C. J. 2006, ApJ, 647, 185

Magorrian, J., Tremaine, S., Richstone, D., et al. 1998, AJ, 115, 2285

Maiolino, R., Salvati, M., Bassani, L., et al. 1998, A\&A, 338, 781

Matt, G., Guainazzi, M., Frontera, F., et al. 1997, A\&A, 325, L13

Matt, G., Fabian, A. C., Guainazzi, M., et al. 2000, MNRAS, 318, 173 
Mayer, L., Kazantzidis, S., Madau, P., et al. 2007, Science, 316, 1874

Mineo, S., Gilfanov, M., \& Sunyaev, R. 2012, MNRAS, 419, 2095

Mitsuishi, I., Yamasaki, N. Y., \& Takei, Y. 2011, ApJL, 742, L31

Nardini, E., Risaliti, G., Watabe, Y., Salvati, M., \& Sani, E. 2010, MNRAS, 405, 2505

Nardini, E., \& Risaliti, G. 2011, MNRAS, 415, 619

Paggi, A., Wang, J., Fabbiano, G., Elvis, M., \& Karovska, M. 2012, ApJ, 756, 39

Palmeri, P., Mendoza, C., Kallman, T. R., Bautista, M. A., \& Meléndez, M. 2003, A\&A, 410, 359

Perlman, E. S., Padgett, C. A., Georganopoulos, M., et al. 2010, ApJ, 708, 171

Pietsch, W., Roberts, T. P., Sako, M., et al. 2001, A\&A, 365, L174

Planck Collaboration, Ade, P. A. R., Aghanim, N., et al. 2016, A\&A, 594, A13

Ranalli, P., Comastri, A., \& Setti, G. 2003, A\&A, 399, 39

Rangwala, N., Maloney, P. R., Glenn, J., et al. 2011, ApJ, 743, 94

Richings, A. J., Fabbiano, G., Wang, J., \& Roberts, T. P. 2010, ApJ, 723, 1375

Risaliti, G., Maiolino, R., \& Salvati, M. 1999, ApJ, 522, 157

Rots, A. H., \& Budavári, T. 2011, ApJS, 192, 8

Sakamoto, K., Wang, J., Wiedner, M. C., et al. 2008, ApJ, 684, 957-977
Sanders, D. B., \& Mirabel, I. F. 1996, ARA\&A, 34, 749

Sanders, D. B., Soifer, B. T., Elias, J. H., et al. 1988, ApJ, 325, 74

Scoville, N. Z., Evans, A. S., Dinshaw, N., et al. 1998, ApJL, 492, L107

Scoville, N., Sheth, K., Walter, F., et al. 2015, ApJ, 800, 70

Scoville, N., Murchikova, L., Walter, F., et al. 2017, ApJ, 836, 66

Siemiginowska, A., Stawarz, Ł., Cheung, C. C., et al. 2007, ApJ, 657, 145

Soifer, B. T., Sanders, D. B., Madore, B. F., et al. 1987, ApJ, 320, 238

Strickland, D. K., \& Heckman, T. M. 2007, ApJ, 658, 258

Suchkov, A. A., Balsara, D. S., Heckman, T. M., \& Leitherer, C. 1994, ApJ, 430,511

Teng, S. H., Veilleux, S., Anabuki, N., et al. 2009, ApJ, 691, 261

Thornton, K., Gaudlitz, M., Janka, H.-T., \& Steinmetz, M. 1998, ApJ, 500, 95

Tunnard, R., Greve, T. R., Garcia-Burillo, S., et al. 2015, ApJ, 800, 25

Van Wassenhove, S., Volonteri, M., Mayer, L., et al. 2012, ApJL, 748, L7

Varenius, E., Conway, J. E., Martí-Vidal, I., et al. 2016, A\&A, 593, A86

Veilleux, S., Rupke, D. S. N., Kim, D.-C., et al. 2009, ApJS, 182, 628-666

Wang, J., Fabbiano, G., Risaliti, G., et al. 2011a, ApJ, 729, 75

Wang, J., Fabbiano, G., Elvis, M., et al. 2011b, ApJ, 736, 62

Wang, J., Fabbiano, G., Elvis, M., et al. 2011c, ApJ, 742, 23

Wang, J., Nardini, E., Fabbiano, G., et al. 2014, ApJ, 781, 55 
X-RAY EMISSION FROM THE NUCLEAR REGION OF ARP 220

Table 1. Summary of Chandra observations of Arp 220.

\begin{tabular}{|c|c|c|c|c|}
\hline \hline OBSID & DATE & $\begin{array}{c}\text { EXPOSURE } \\
(\mathrm{ks})\end{array}$ & PI & $\begin{array}{c}0.5-8 \text { keV COUNTS } \\
\text { (central 5” region) }\end{array}$ \\
\hline 00869 & 2000 -Jun-24 & 57 & Clements & 583 \\
16092 & $2014-$ Arp-30 & 170 & Fabbiano & 1390 \\
16093 & 2014-Jun-24 & 67 & Fabbiano & 543 \\
\hline \hline
\end{tabular}

Table 2. Best fit parameters for the extraction regions considered in the main text.

\begin{tabular}{|c|c|c|c|c|c|c|c|c|c|}
\hline & \multicolumn{3}{|c|}{$\mathrm{W}$ region } & \multicolumn{3}{|c|}{ E region } & \multicolumn{3}{|c|}{ Central 4.5" } \\
\hline Band & \multicolumn{9}{|c|}{ Net counts (error) } \\
\hline $3-8 \mathrm{keV}$ & \multicolumn{3}{|c|}{$75(9)$} & \multicolumn{3}{|c|}{$221(15)$} & \multicolumn{3}{|c|}{$659(26)$} \\
\hline $6-7 \mathrm{keV}$ & \multicolumn{3}{|c|}{$15(3)$} & \multicolumn{3}{|c|}{$41(6)$} & \multicolumn{3}{|c|}{$90(10)$} \\
\hline $6-6.4 \mathrm{keV}$ & \multicolumn{3}{|c|}{$6(2)$} & \multicolumn{3}{|c|}{$11(3)$} & \multicolumn{3}{|c|}{$25(5)$} \\
\hline 6.4-6.7 keV & \multicolumn{3}{|c|}{$8(3)$} & \multicolumn{3}{|c|}{$27(5)$} & \multicolumn{3}{|c|}{$55(7)$} \\
\hline Model parameter & \multicolumn{9}{|c|}{ Best-fit value } \\
\hline Spectral model (Power-law + lines) & (a) & (b) & (c) & (a) & (b) & (c) & (a) & (b) & (c) \\
\hline$\Gamma_{P L}$ & $3.40^{*}$ & $3.40^{*}$ & $3.40^{*}$ & $2.39^{*}$ & $2.39^{*}$ & $2.39^{*}$ & $3.12^{*}$ & $3.12^{*}$ & $3.12^{*}$ \\
\hline$F_{P L}\left(10^{-4} \mathrm{~cm}^{-2} \mathrm{~s}^{-1} \mathrm{keV}^{-1}\right)$ & $18.30_{-1.72}^{+2.27}$ & $18.97_{-1.93}^{+2.07}$ & $18.06_{-1.90}^{+2.04}$ & $4.48_{-0.67}^{+0.40}$ & $5.56_{-0.51}^{+0.55}$ & $4.47_{-0.50}^{+0.38}$ & $38.65_{-3.42}^{+2.43}$ & $44.42_{-2.26}^{+2.37}$ & $38.91_{-2.27}^{+2.29}$ \\
\hline$E_{F e-K}(\mathrm{keV}$, rest-frame $)$ & $6.61_{-0.07}^{+0.06}$ & $6.4^{*}$ & $6.4^{*}$ & $6.67_{-0.03}^{+0.03}$ & $6.4^{*}$ & $6.4^{*}$ & $6.65_{-0.02}^{+0.02}$ & $6.4^{*}$ & $6.4^{*}$ \\
\hline$F_{F e-K}\left(10^{-7} \mathrm{~cm}^{-2} \mathrm{~s}^{-1}\right)$ & $4.01_{-1.59}^{+1.97}$ & $2.39_{-1.27}^{+1.59}$ & $1.16_{-1.09}^{+1.42}$ & $9.43_{-1.91}^{+2.17}$ & $1.78_{-1.27}^{+1.51}$ & $<0.86$ & $11.92_{-2.07}^{+2.21}$ & $<5.01$ & $<0.88$ \\
\hline$L_{F e-K}\left(10^{40} \mathrm{erg} \mathrm{s}^{-1}\right)$ & $0.32_{-0.14}^{+0.13}$ & $0.19_{-0.10}^{+0.11}$ & $0.09_{-0.07}^{+0.07}$ & $0.76_{-0.15}^{+0.17}$ & $0.14_{-0.08}^{+0.11}$ & $<0.04$ & $0.96_{-0.17}^{+0.17}$ & $<0.29$ & $<0.06$ \\
\hline$E W_{F e-K}(\mathrm{keV})$ & $1.22_{-0.53}^{+0.57}$ & $0.67_{-0.36}^{+0.43}$ & $0.35_{-0.28}^{+0.29}$ & $1.76_{-0.43}^{+0.45}$ & $0.27_{-0.15}^{+0.23}$ & $<0.11$ & $1.02_{-0.19}^{+0.19}$ & $<0.27$ & $<0.06$ \\
\hline$E_{F e-K}(\mathrm{keV}$, rest-frame $)$ & - & - & $6.7^{*}$ & - & - & $6.7^{*}$ & - & - & $6.7^{*}$ \\
\hline$F_{F e-K}\left(10^{-7} \mathrm{~cm}^{-2} \mathrm{~s}^{-1}\right)$ & - & - & $3.33_{-1.58}^{+1.94}$ & - & - & $9.62_{-1.99}^{+2.16}$ & - & - & $11.86_{-2.03}^{+2.22}$ \\
\hline$L_{F e-K}\left(10^{40} \mathrm{erg} \mathrm{s}^{-1}\right)$ & - & - & $0.27_{-0.13}^{+0.15}$ & - & - & $0.78_{-0.19}^{+0.18}$ & - & - & $0.96_{-0.08}^{+0.12}$ \\
\hline$E W_{F e-K}(\mathrm{keV})$ & - & - & $1.04_{-0.47}^{+0.60}$ & - & - & $1.80_{-0.46}^{+0.48}$ & - & - & $0.97_{-0.08}^{+0.13}$ \\
\hline C-stat (d.o.f.) & $34.9(29)$ & $47.0(30)$ & $42.3(29)$ & $86.3(66)$ & $137.5(67)$ & $85.5(66)$ & $288.1(211)$ & $352.2(212)$ & $292.3(211)$ \\
\hline$L_{2-10 \mathrm{keV}}\left(10^{40} \mathrm{erg} \mathrm{s}^{-1}\right)$ & $1.99_{-0.23}^{+0.20}$ & $1.91_{-0.23}^{+0.23}$ & $2.01_{-0.23}^{+0.26}$ & $3.22_{-0.32}^{+0.31}$ & $3.08_{-0.29}^{+0.31}$ & $3.23_{-0.33}^{+0.32}$ & $6.81_{-0.39}^{+0.39}$ & $6.56_{-0.43}^{+0.44}$ & $7.13_{-0.30}^{+0.35}$ \\
\hline Model parameter & \multicolumn{9}{|c|}{ Best-fit value } \\
\hline Spectral model (APEC) & \multicolumn{3}{|c|}{ (d) } & \multicolumn{3}{|c|}{ (d) } & \multicolumn{3}{|c|}{ (d) } \\
\hline$N_{H}\left(10^{22} \mathrm{~cm}^{-2}\right)$ & $5.63_{-1.07}^{+3.20}$ & \multicolumn{2}{|c|}{$5.65_{-1.32}^{+1.89}$} & $5.83_{-0.69}^{+0.90}$ & \multicolumn{2}{|c|}{$4.75_{-0.74}^{+0.74}$} & $2.42_{-0.51}^{+0.79}$ & \multicolumn{2}{|c|}{$2.28_{-0.61}^{+1.08}$} \\
\hline$k T(\mathrm{keV})$ & $5.58_{-2.27}^{+5.34}$ & \multicolumn{2}{|c|}{$5.44_{-1.51}^{+2.71}$} & $5.18_{-1.05}^{+0.79}$ & \multicolumn{2}{|c|}{$5.53_{-0.70}^{+1.11}$} & $5.30_{-1.14}^{+1.26}$ & \multicolumn{2}{|c|}{$5.21_{-1.02}^{+1.01}$} \\
\hline $\operatorname{EM}\left(10^{-4} \mathrm{~cm}^{-2} \mathrm{~s}^{-1}\right)$ & $2.95_{-0.76}^{+1.49}$ & \multicolumn{2}{|c|}{$2.89_{-0.72}^{+1.88}$} & $4.58_{-0.72}^{+1.29}$ & \multicolumn{2}{|c|}{$2.31_{-0.42}^{+0.65}$} & $7.42_{-0.94}^{+1.22}$ & \multicolumn{2}{|c|}{$6.45_{-1.15}^{+1.85}$} \\
\hline $\mathrm{Z}$ & $1^{*}$ & 1.14 & $\begin{array}{r}-0.75 \\
-0.44\end{array}$ & $1^{*}$ & 4.14 & $\begin{array}{r}2.30 \\
-1.29\end{array}$ & $1^{*}$ & 1.6 & $\begin{array}{r}+0.59 \\
-0.52\end{array}$ \\
\hline C-stat (d.o.f.) & $111.6(40)$ & 111. & (39) & $261.6(94)$ & 247. & (93) & $306.8(310)$ & 305. & (309) \\
\hline$L_{0.5-8 \mathrm{keV}}\left(10^{40} \mathrm{erg} \mathrm{s}^{-1}\right)$ & $3.85_{-0.62}^{+0.70}$ & 3.85 & $\begin{array}{l}+1.51 \\
-1.41 \\
\end{array}$ & $5.89_{-0.89}^{+0.86}$ & 5.42 & $\begin{array}{r}+1.52 \\
-1.62 \\
\end{array}$ & $9.59_{-4.46}^{+4.96}$ & 9.6 & $\begin{array}{r}+3.55 \\
-3.15 \\
\end{array}$ \\
\hline
\end{tabular}




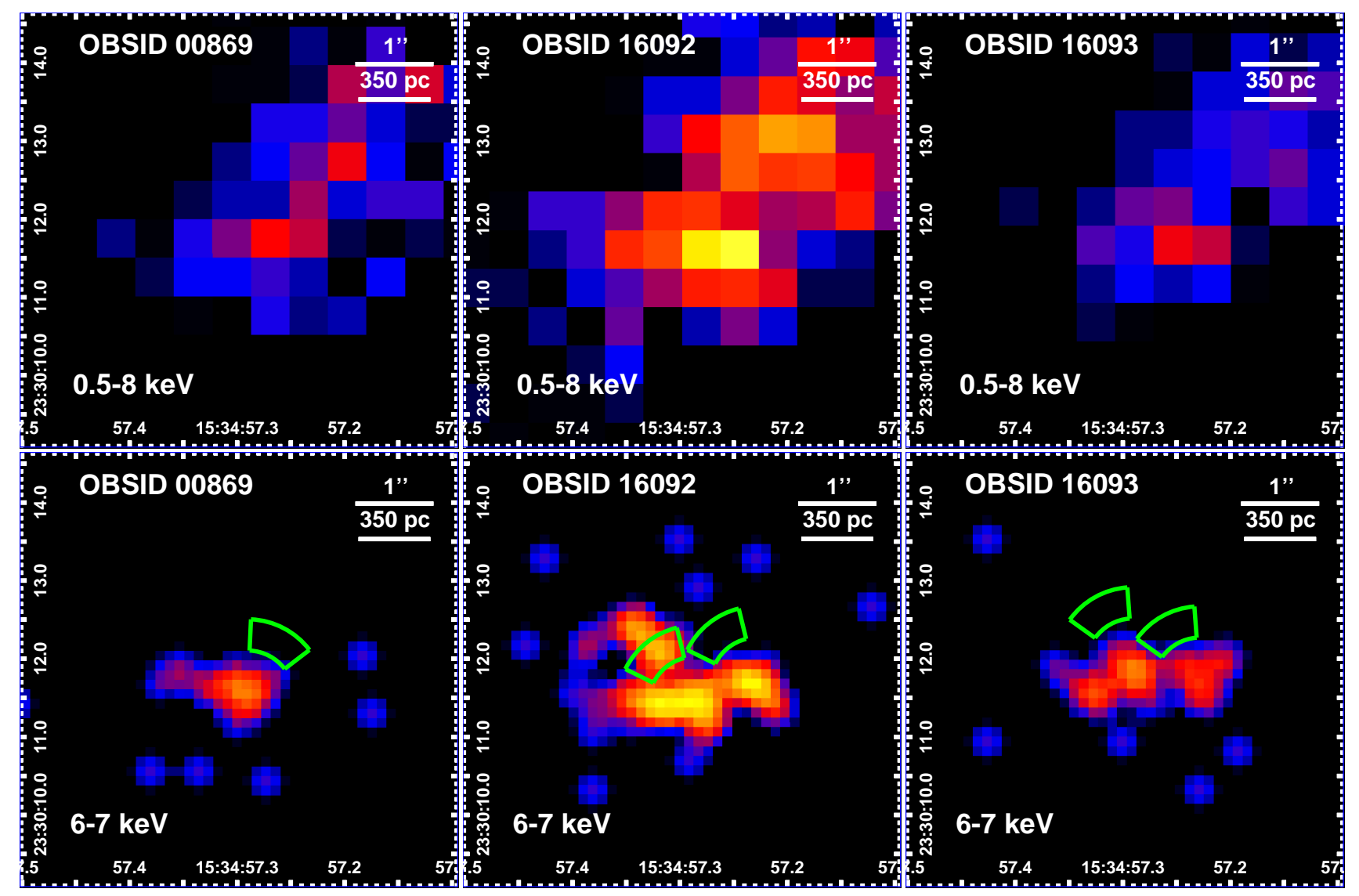

Figure 1. (Top panels) Broad band 0.5-8 keV images of the central 5" region of Arp 220 for three Chandra-ACIS observations reprojected on OBSID 16092, with native 0.492" pixel size. (Bottom panels) Same as top panels but in narrow band 6-7 keV. Images are presented with sub-pixel binning ( $1 / 4$ of the native pixel size) and 3 X3 pixel FWHM gaussian filter smoothing. Green regions show the location of the Chandra PSF artifacts for the two nuclei.

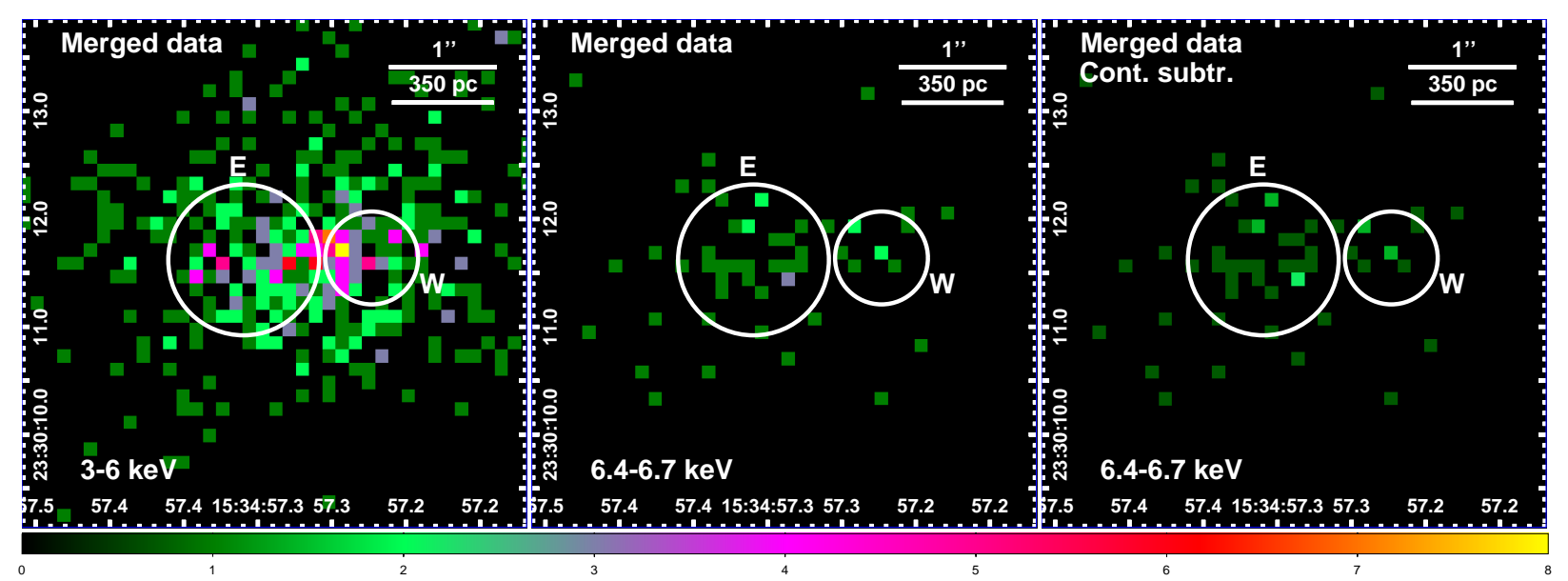

Figure 2. From left to right: merged Chandra-ACIS 3-6 and 6.4-6.7 keV images, indicative of continuum and Fe xxv line emission, respectively. In the right panel we show the 6.4-6.7 keV band image after continuum (3-6 keV) subtraction. Images are presented with sub-pixel binning $1 / 4$ of the native pixel size. The regions of spectral extraction considered in Section 2.2 are shown as white circles. 


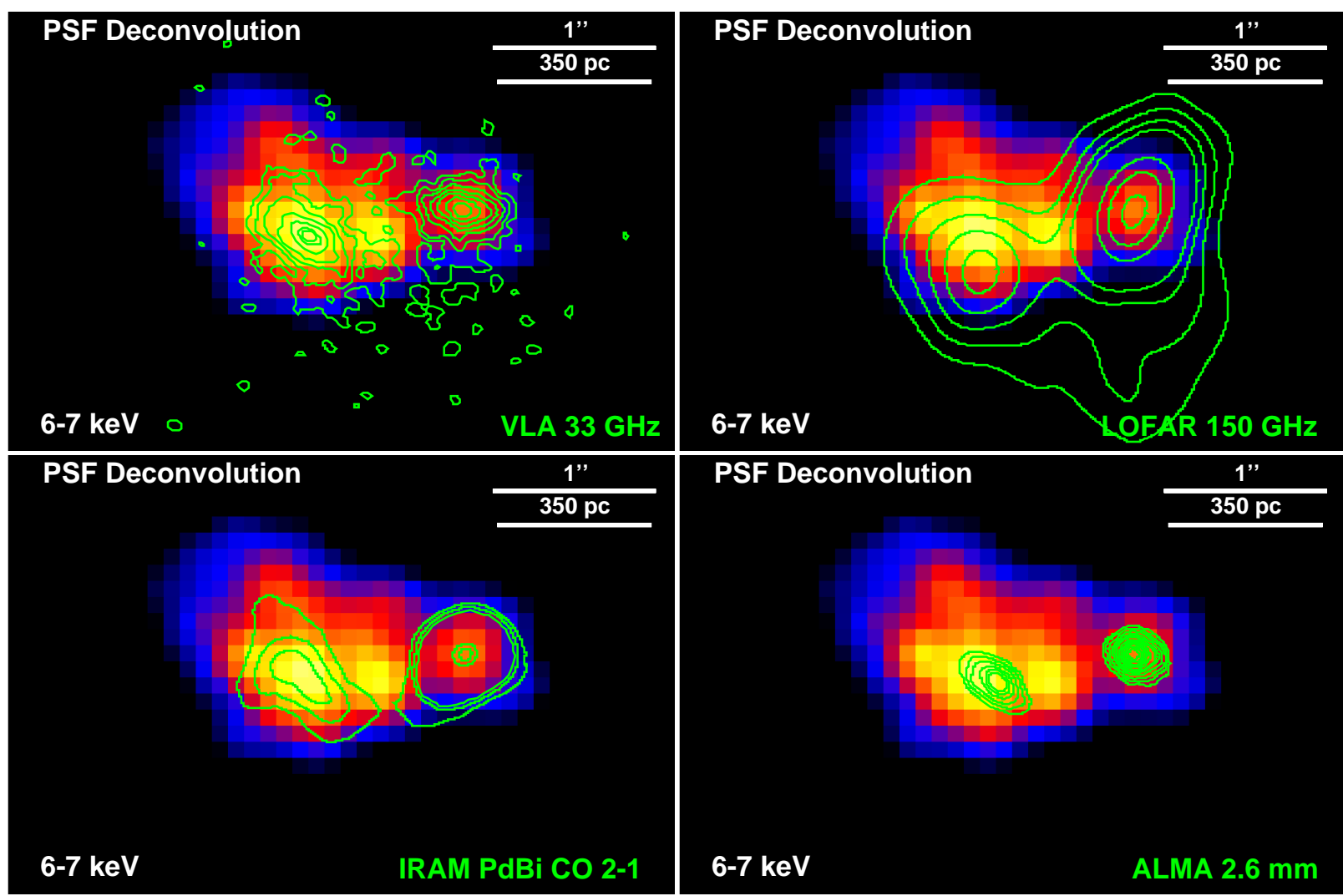

Figure 3. Merged, PSF-deconvolved Chandra-ACIS 6-7 keV image with sub-pixel binning $1 / 5$ of the native pixel size and 3 pixel FWHM gaussian filter smoothing, with superimposed (from top left to bottom right) contours from $33 \mathrm{GHz}$ VLA data (Barcos-Muñoz et al. 2015), the $150 \mathrm{MHz}$ continuum and CO 2-1 IRAM PdBi data (Varenius et al. 2016), and the $2.6 \mathrm{~mm}$ continuum ALMA data (Scoville et al. 2017), registered as discussed in the main text.
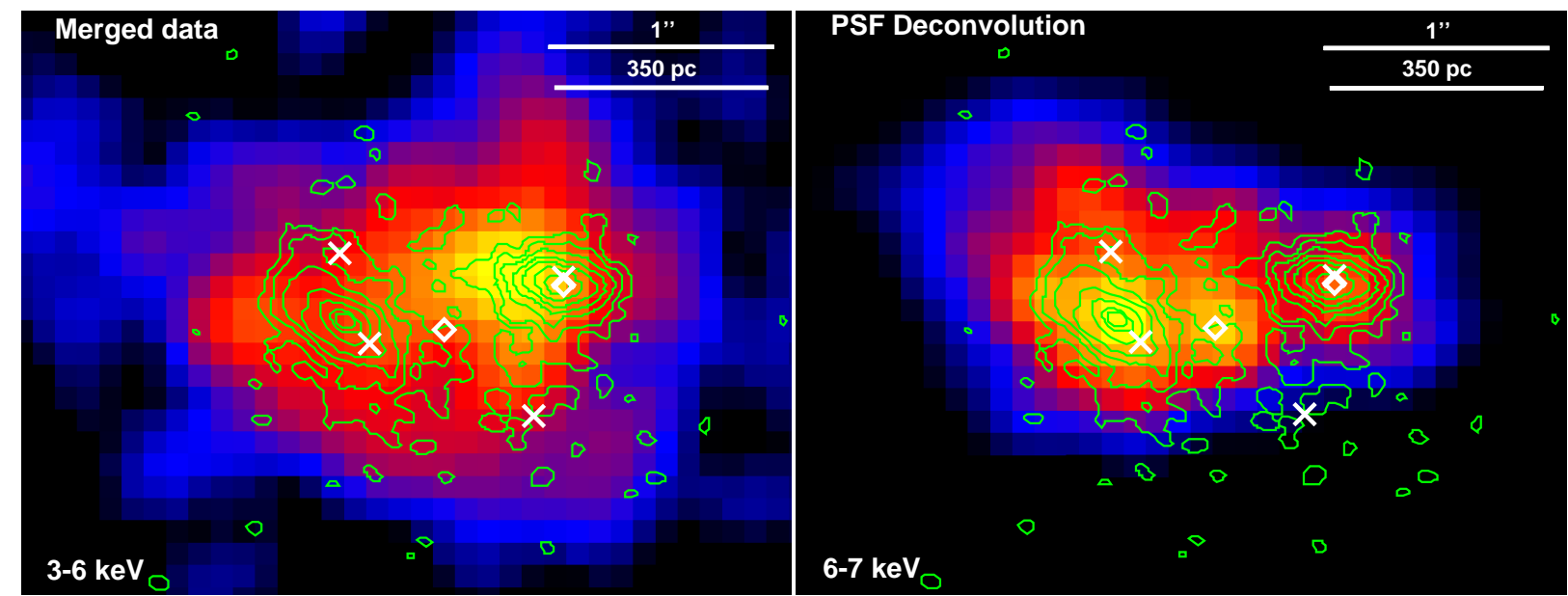

Figure 4. Merged Chandra-ACIS 3-6 keV (left panel) and 6-7 keV PSF-deconvolved (right panel) images, indicative of continuum and Fe line emission, respectively. Images are presented with sub-pixel binning $1 / 5$ of the native pixel size and 3 pixel FWHM gaussian filter smoothing. Green lines represent $33 \mathrm{GHz}$ VLA contours as in Figure 3, white crosses represent the NIR sources from Scoville et al. (1998) and white diamonds represent the X-ray sources from Clements et al. (2002). 

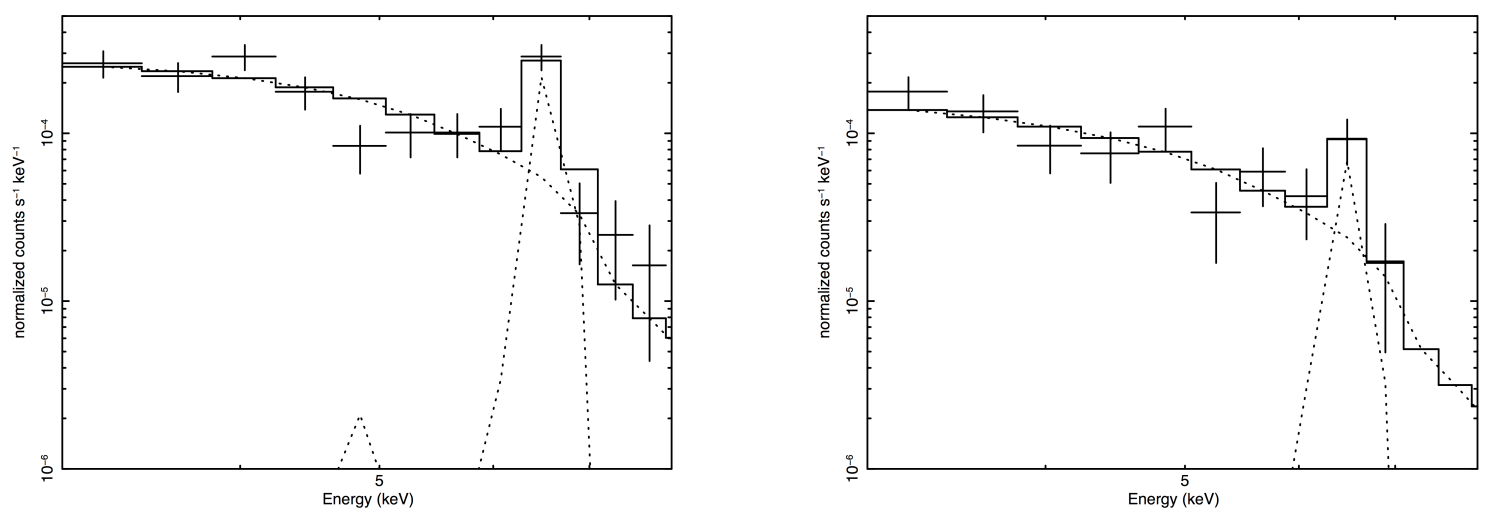

Figure 5. Best fit to spectra extracted from regions shown in Figure 2 from E region (left panel) and W region (right panel). For clarity only the reflection component and $\mathrm{Fe}-\mathrm{K}$ line are shown with a dotted line.
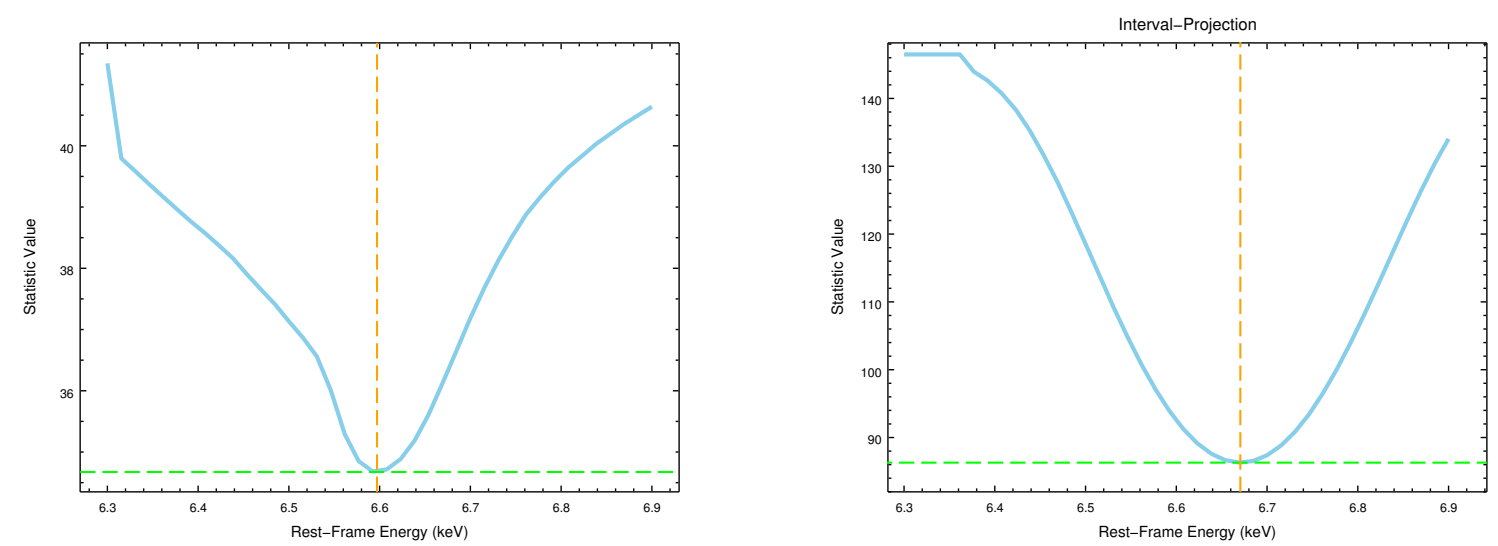

Figure 6. Value of the fit statistic of model a as function of the rest-frame line energy in W (left panel) and E (right panel) nuclei.

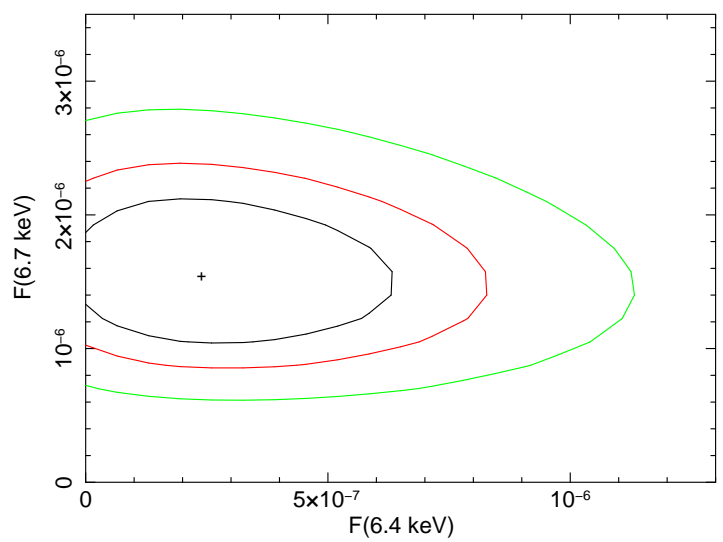

Figure 7. Contour plot for the relative flux of the 6.4 and $6.7 \mathrm{keV}$ lines in a fit where both rest-frame energies are fixed in the XMM-Newton data form the nuclear $(\mathrm{E}+\mathrm{W})$ region. The contours represent a probability of $68 \%, 90 \%$ and $99 \%$. Even if a solution with a single $6.7 \mathrm{keV}$ line is the more likely, a contribution of up to $40 \%$ by a neutral line is compatible with the data at a $90 \%$ confidence level. 

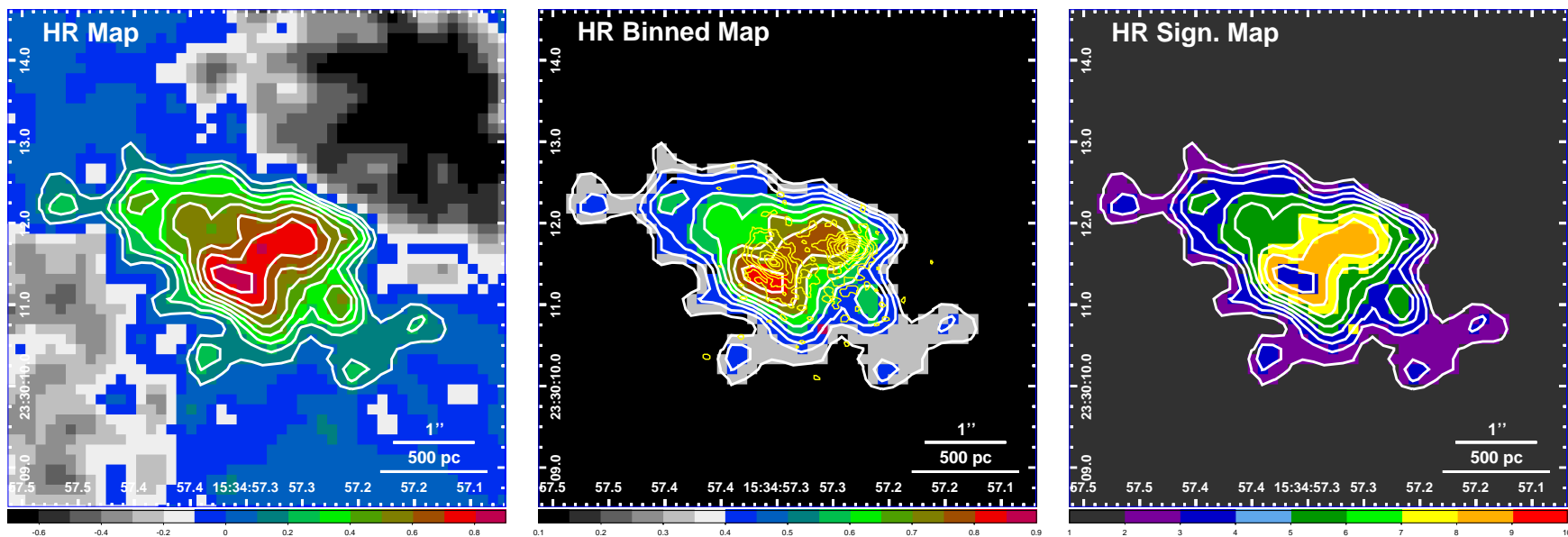

Figure 8. (Left Panel) HR map for the central region of Arp 220 with sub-pixel binning 1/4 of the native pixel size and a $3 X 3$ pixel FWHM gaussian filter smoothing. The white contours indicate levels of HR from 0.1 to 0.8 with increments of 0.1. (Central Panel) Same as left panel, but with the map binned using the contour levels. In yellow we show the $33 \mathrm{GHz}$ VLA contours. (Right Panel) Significance map of the HR binned map.
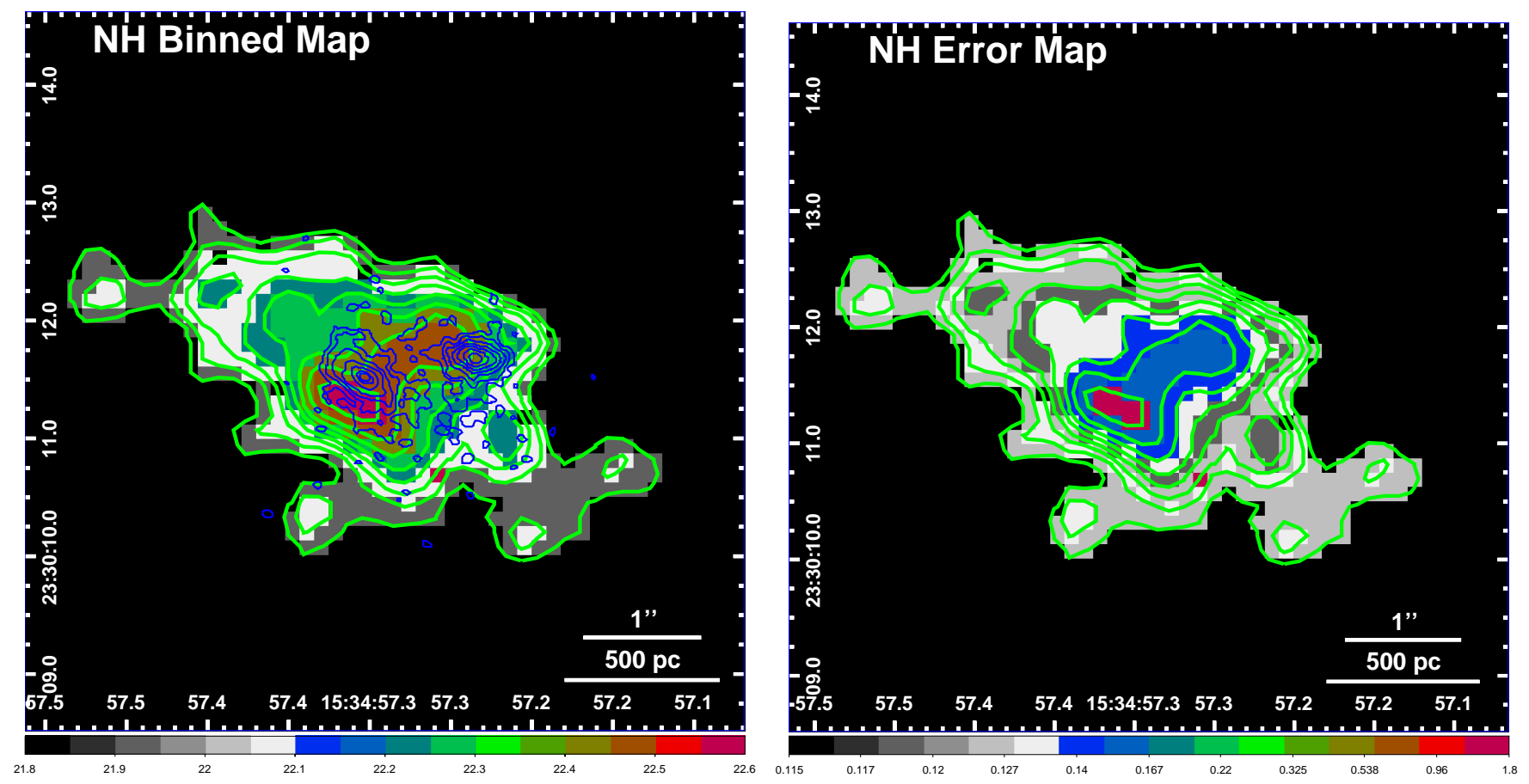

Figure 9. (Left Panel) Logarithmic map of the absorption column as evaluated from the HR map presented in the central panel of Figure 8 assuming a power-law spectrum with 1.8 slope. In blue are overplotted the $33 \mathrm{GHz}$ VLA contours. (Right Panel) Map of the error on $\log \left(N_{H} / \mathrm{cm}^{2}\right)$ evaluated from the uncertainty on the HR. 